\title{
Ergotherapie
}

\section{So klappt's auch mit der Motivation}

\author{
KLIENTEN-COACHING Um nachhaltig etwas zu verändern, reicht eine Therapieeinheit \\ pro Woche meist nicht aus. Aktiv werden und auch zu Hause üben, lautet deshalb die \\ Devise. Wenn das immer so einfach wäre! In der Praxis haben es Ergotherapeuten \\ nämlich nicht nur mit fleißigen und motivierten Klienten zu tun, da sind auch ein paar \\ lustlose und unentschlossene dabei. Zum Glück gibt's für jeden die passende Strategie.
}

M it 45 Minuten Therapie pro Woche ist es nicht getan. Eigenübungen und der Transfer in den Alltag sind wichtige Bestandteile der Behandlung. Sie gelten als entscheidender Beitrag zur nachhaltigen Genesung. Doch jeder Klient verhält sich anders von lustlos über bedingt motiviert bis hin zu ehrgeizig. Da wäre es manchmal schön, wenn man Motivation in einen Menschen hineinpumpen könnte wie Treibstoff in einen Tank. Leider funktioniert das in der Praxis - wenn überhaupt - nur recht kurzfristig. Die Vorschläge, Eigenübungen auch außerhalb der Therapie umzusetzen, verhallen.

Behandlungseinheiten mit solchen Klienten führen häufig zu Frusterlebnissen, manchmal zu Streitgesprächen und gelegentlich zu resignierten Therapeuten: „Bei Herrn Schmidt erwähne ich schon gar nicht mehr, dass er seine Hausaufgaben machen soll, es hat ja doch keinen Sinn. “ Eine elegantere Möglichkeit ist, die Lösung beim Klienten zu suchen. Denn: Die Motivation für Eigenaktivität muss er selbst entwickeln. Hier greift ein Konzept zum KlientenManagement, das Ansätze aus der Gesundheitspsychologie mit Coaching-Interventionen kombiniert: das Klienten-Coaching.

Das Motivationsstadium eines Klienten bestimmen > Um es einem Klienten schmackhaft machen zu können, dass er seine Übungen auch zu Hause umsetzt, hilft die Erkenntnis, dass ein Mensch verschiedene Stadien durchwandert, bevor er sein Verhalten ändert. Zum Gesundheitsverhalten existieren mehrere Modelle, die eine relevante Gemeinsamkeit haben: Sie beschreiben alle eine motivationale, volitional-inaktive und volitional-aktive Phase [1]. Das heißt, ein anfangs lustloser Mensch hat nicht unbedingt immer ein Motivationsproblem. Schreitet er im Änderungsprozess weiter fort, motiviert er sich mit der Zeit, setzt die Übung aber möglicherweise noch nicht um, weil ihm die willentliche Kontrolle fehlt. Eventuell verändert er sein Verhalten schließlich tatsächlich und wird aktiv, gibt aber beispielsweise durch einen Rückfall schnell wieder auf.

Daraus ergeben sich vier verschiedene Stadien: Idee und Motivation, Planung und Umsetzung, aktiv werden sowie Coaching und Befähigung (IPaC) (๑ Abb. 1).
Ambivalente Klienten durch Hinterfragen aktivieren > Ein Klient, der sich im ersten Stadium auf dem Weg zur Verhaltensänderung befindet, hat noch keine Intention gebildet. Das heißt, entweder hat er noch gar keine Idee, wie er sein Vorhaben umsetzen könnte, oder er will gar nichts ändern. Hier ist die Ergotherapeutin herausgefordert, ihn zu motivieren. Aber wie?

Häufig sind Klienten nicht komplett unmotiviert, sondern ambivalent. Erkennen kann man dies an einem „Aber“. Ein Klient möchte zum Beispiel häufiger spazieren gehen, aber das Sofa ist immer so bequem. Dieses innere Wechselspiel kann ihm die Therapeutin anhand von Fragen veranschaulichen [5, 6, 7]. Formuliert sie diese offen, kann sie Unterschiede verdeutlichen, andere Sichtweisen ermöglichen, die Aufmerksamkeit auf die gewünschte (Lösungs-)Richtung lenken und damit letztendlich seine Bereitschaft zur Veränderung erhöhen. Auf diese Weise regt die Therapeutin den Klienten zum Reflektieren an. Wichtig dabei ist, dass sie ihm dafür genügend Zeit gibt, ihm aktiv zuhört und ihn seine eigene Ambivalenz durch das Widerspiegeln des Gesagten erforschen lässt. Dadurch kann er bereits vorhandene Argumente, die für eine Veränderung sprechen, verstärkt wahrnehmen. Je deutlicher er sich diesen Unterschied zwischen tatsächlichem und erwünschtem Zustand vor Augen führt, desto stärker wächst seine innere Motivation.

Klienten brauchen einen wichtigen Grund und Zuversicht, um etwas ändern zu wollen > In der Praxis hat sich das Hinterfragen zweier Kriterien, die die Eigenmotivation beeinflussen, bewährt [3]. Einerseits, wie wichtig dem Klienten eine Verhaltensänderung erscheint, und andererseits, wie zuversichtlich er ist, beim Ändern seines Verhaltens erfolgreich zu sein.

Um diese beiden Kriterien einschätzen zu können, bieten sich Ratingskalen an (๑ Abb. 2). Einigen Klienten hilft es, diese Fragen schriftlich zu beantworten. In der Therapie kann man die Punkte jedoch auch mündlich abfragen, zum Beispiel mit: „Wie wichtig ist es Ihnen auf einer Skala von 0 bis 10, Ihr Verhalten zu ändern, wenn 0 gar nicht wichtig und 10 extrem wichtig ist?“ Oder: „Wie zuversichtlich schätzen Sie sich auf einer Skala von 0 bis 10 ein, 


\section{Ergotherapie}

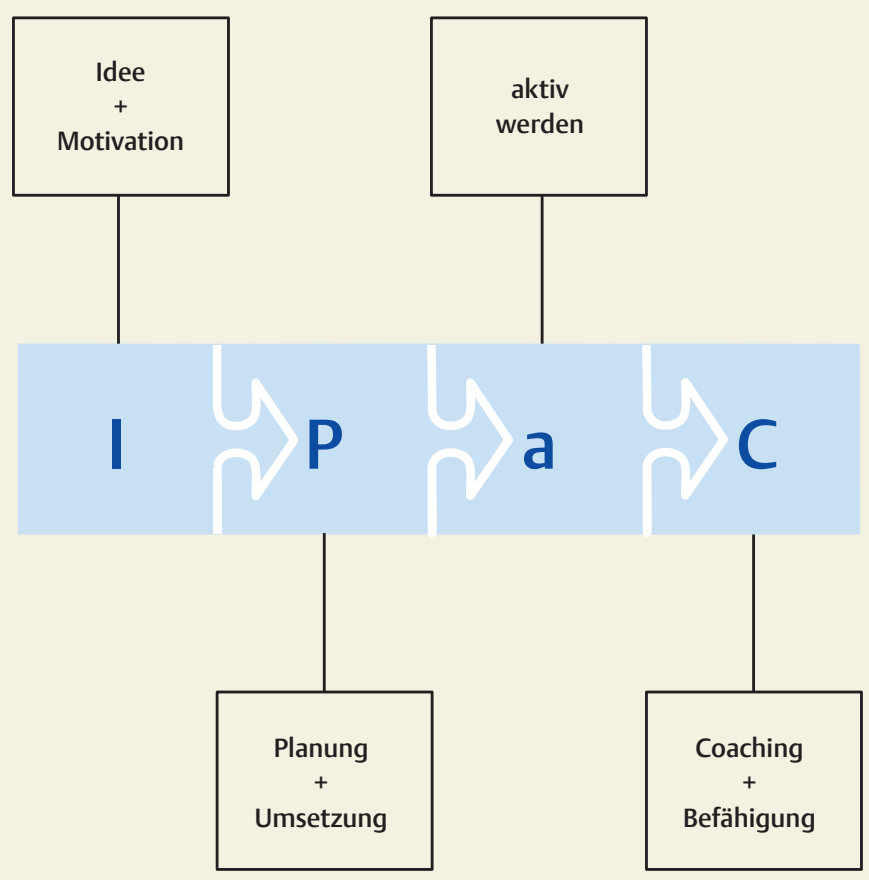

Abb. 1 Ein Klient durchläuft vier Stadien auf dem Weg zur Verhaltensänderung: von der Idee, eine Übung auch zu Hause durchzuführen, bis hin zur Befähigung, dies auch tatsächlich zu tun.

\begin{tabular}{|c|c|c|c|c|c|c|c|c|c|c|}
\hline \multicolumn{10}{|c|}{ Wie wichtig ist es für Sie ...? } \\
\hline 0 & 1 & 2 & 3 & 4 & 5 & 6 & 7 & 8 & 9 & 10 \\
\hline $\begin{array}{l}\text { Gar nicht } \\
\text { wichtig }\end{array}$
\end{tabular}

\begin{tabular}{|c|c|c|c|c|c|c|c|c|c|c|}
\hline \multicolumn{10}{|c|}{ Wie zuversichtlich sind Sie ... umzusetzen? } \\
\hline 0 & 1 & 2 & 3 & 4 & 5 & 6 & 7 & 8 & 9 & 10 \\
\hline $\begin{array}{c}\text { Gar nicht } \\
\text { zuver- } \\
\text { sichtlich }\end{array}$
\end{tabular}

Abb. 2 Ratingskalen wie diese machen deutlich, wie wichtig ein Verhalten für den Klienten ist bzw. wie zuversichtlich er ist, dieses Verhalten selbstständig umzusetzen. Das regt nicht nur die Selbstreflexion an, sondern signalisiert der Therapeutin auch, mit welchem Motivationstyp sie es zu tun hat. 


\section{Ergotherapie}

dass Sie die Übung auch alleine umsetzen können, wenn 0 gar nicht zuversichtlich und 10 extrem zuversichtlich ist?" Kombiniert die Therapeutin diese Einschätzung mit einer Reflexion, ermöglicht sie damit dem Klienten, eine Änderungstendenz aufzubauen.

Klienten folgen: die Therapeutin als Gefährtin > Die Art und Weise, wie sich die Therapeutin verhält und wie sie kommuniziert, hat einen nicht zu unterschätzenden Einfluss auf die Veränderungsbereitschaft und den Veränderungsprozess. Empathie, Akzeptanz und Kongruenz gelten als Basis jeder therapeutischen Interaktion ( $\odot$ „Empathie“, S. 34) [2]. Setzt die Therapeutin diese erfolgreich um, fühlt sich der Klient verstanden und aufgehoben. Ein möglicher Widerstand bleibt gering oder entsteht erst gar nicht.

Darüber hinaus unterscheidet man die drei Kommunikationsstile „Folgen“, „Führen“ und „Begleiten“, welche eine Therapeutin bewusst und je nach Bedarf wählen kann [3, 4].

Folgen bedeutet für die Ergotherapeutin, dem Klienten zu signalisieren, dass sie für ihn da ist. Sie hört ihm gut zu und unterstützt ihn dabei, seine Gefühle anzusprechen. Das kann ihn entlasten und mögliche Unklarheiten beseitigen. Dennoch ist das Ganze die Reise des Klienten, die er in seinem eigenen Tempo geht. Die Therapeutin nimmt dabei die Rolle der Gefährtin ein, die nicht den Weg vorschlägt, sondern zur Seite steht und folgt. Dieser Stil ist vor allem dann sinnvoll, wenn der Klient eine emotional besonders belastende Situation verarbeiten muss. Das können sowohl schlechte Nachrichten bezüglich seiner Krankheit sein oder auch die kürzlich angeordnete Kurzarbeit mit der Sorge um seinen Arbeitsplatz.

Dem Klienten zu Beginn der Behandlung zu folgen und ihn dort abzuholen, wo er steht, erleichtert es der Ergotherapeutin, die Symptome aus seinem Blickwinkel sowie sein Denken und sein Fühlen zu verstehen.

Klienten führen: die Therapeutin als Kapitän > Beim Führen hat die Therapeutin eine klare Meinung darüber, was dem Klienten gut tut. Sie kann das Problem erklären und schlägt Lösungen vor. Hierbei ist sie der Kapitän, der den Kurs bestimmt. Das Führen basiert auf Fachwissen und Autorität. Man kann es bei Klienten einsetzen, die fachlichen Rat und/oder externe Motivationsquellen benötigen.

Zu bedenken gilt: Nur etwa 20 Prozent aller Klienten verändern ihr Verhalten aufgrund von sachlichen Informationen [14]. Es kann sogar sein, dass genau das Gegenteil passiert und der Klient mit Widerstand reagiert. Da hilft es dann auch nicht, Druck auszuüben. Der würde das Ganze nur noch verstärken.

INTERNET

\section{Gesundheitscoaching}

Wer sich für das Konzept hinter „IPaC“ interessiert und mehr über Gesundheitscoaching erfahren möchte, der findet weitere Infos im Internet unter www.emota.de.
Klienten begleiten: die Therapeutin als Geburtshelferin > Der Kommunikationsstil des Begleitens ist eine Mischung aus Folgen und Führen. Die Therapeutin hört gut zu, ist empathisch und versteht das Problem. Sie fragt den Klienten nach den Optionen, die er schon angedacht hat, und wägt gemeinsam mit ihm Vor- und Nachteile ab. Dabei kann sie ihm auch einige praktische Tipps anbieten. Die Person, die entscheidet, bleibt aber letztendlich der Klient. Die Therapeutin unterstützt mit diesem begleitenden Stil als „Geburtshelferin" seine Entscheidung. Legt sich der Klient dann selbst auf ein Ziel fest, akzeptiert er es besser und bindet sich emotional stärker daran. Auf diese Weise kann man einen möglichen Widerstand umgehen und die Adhärenz steigern. Besonders im Stadium „Idee und Motivation“ ist dieser Stil sehr effektiv (๑ Abb. 1, S. 21).

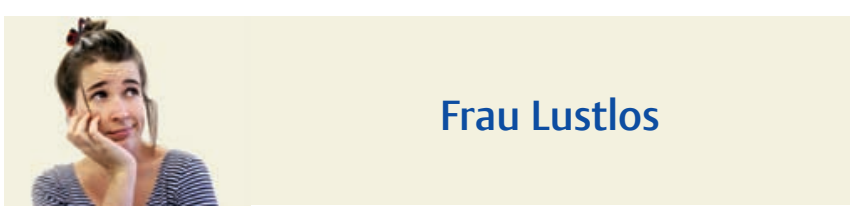

Wenn die Motivation fehlt, ist aktives Reflektieren gefragt > Frau Lustlos kommt mit Sensibilitätsstörungen in der Hand zu Lisa in die Praxis. Die Ergotherapeutin merkt schnell, dass die neue Klientin nicht bereit ist, auch außerhalb der Therapie zu üben. Damit befindet sie sich im ersten Stadium „Idee und Motivation“. Auf der Wichtigkeitsskala gibt sie eine Drei an. Das bedeutet, dass ihr das Warum der Eigenaktivität noch nicht klar ist. Was kann Lisa tun?

Eine Möglichkeit wäre, Frau Lustlos zu fragen, warum sie diesen und keinen niedrigeren Wert gewählt hat. Die Antwort würde den Fokus automatisch auf die Gründe lenken, die die Wichtigkeit steigern. Ähnliche Reaktionen erlaubt die Frage: „Was müsste passieren, damit Sie sich für einen höheren Wert entscheiden?“ Lisa sollte auch den Zwiespalt von Frau Lustlos im Hinterkopf haben, um das Für und Wider aus verschiedenen Perspektiven abwägen zu können. Auf diese Weise kann sie die Nachteile des Ist-Zustandes, also des Nicht-üben-Wollens, durch Fragen hervorbringen: „Was, glauben Sie, wird geschehen, wenn Sie nichts verändern?“ Nach den Vorteilen einer aktiven Veränderung kann Lisa direkt fragen oder auch etwas weiter ausholen, um die Werte und Ziele dahinter zu erforschen: „Was sind Ihrer Ansicht nach die Hauptgründe für eine Veränderung?“ oder „Was ist Ihnen an Ihrer Genesung am wichtigsten?“ oder „Was sind Ihre schlimmsten Befürchtungen, was passieren könnte, wenn Sie nicht aktiv werden?“.

Die Wichtigkeit wird unter anderem über den Glauben oder das Gefühl des Klienten, durch sein Üben etwas zu erreichen, gesteigert. Lisa kann diese erfragen mit „Wenn Sie sich entscheiden würden, selbst aktiv zu werden, was würden Sie sich davon versprechen?“. Hier kann sie unterstützend wirken, denn Klienten wissen schließlich nicht alles über Wirkungsweisen und brauchen deshalb Informationen über die Übungseffekte.

Die Fragen zur Zuversicht kann Lisa dann ähnlich wie bei der Wichtigkeit stellen. Frau Lustlos schätzt sich mit einer Vier ein. Lisa hilft ihr dabei, auch das Wie der Eigenübung bzw. der Veränderung zu verstehen, und reagiert mit: „Warum haben Sie sich keine Eins 
gegeben?“ Hilfreich sind auch: „Was würde Ihnen helfen, auf eine höhere Zahl zu kommen?“ oder „Wie kann ich Sie unterstützen, auf der Skala nach oben zu wandern?“ [3]. Ist Frau Lustlos noch sehr unsicher, nutzen Rückblicke auf vergangene Erfolge. Vielleicht gab es ähnliche Situationen in ihrem Leben, von denen sie jetzt profitieren kann [8]. Direkt nach den Ressourcen zu suchen, die den Klienten unterstützen, gelingt durch: „Wer bzw. was kann Sie unterstützen?" Lisa darf als Expertin natürlich auch Tipps und Informationen weitergeben. Als Angebot und nicht als einzig möglicher Weg präsentiert, wird Frau Lustlos diese eher annehmen.

Sobald die Klientin überzeugt ist und Eigenübungen für wichtig erachtet, sollte sich die Frage nach dem folgenden Schritt anschließen. Wie geht es jetzt konkret weiter, um den Sprung in das nächste Stadium zu schaffen?

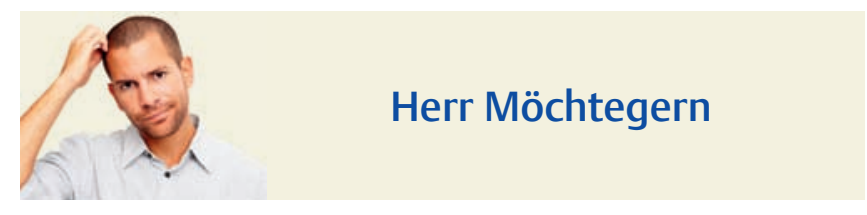

Wenn der Wille da und das Fleisch schwach ist, muss ein Vertrag her > Dieselbe Erkrankung, ein anderer Klient, ein anderes Bild. Herr Möchtegern hat ebenfalls seit geraumer Zeit Sensibilitätsstörungen in der Hand. Ergotherapeutin Lisa erkennt eine ähnliche Ursache wie bei Frau Lustlos, sieht jedoch, dass dieser Klient eine ganz andere Motivationslage aufweist. Er scheint eine feste Absicht gebildet zu haben und betont das auch in jeder Behandlung. Dennoch muss Lisa feststellen, dass er, obwohl hoch motiviert, seine Aufgaben nicht regelmäßig durchführt. Er befindet sich im Stadium „Planung und Umsetzung“. Die Motivation weiter zu steigern, ist hier nicht mehr effektiv. Lisa sollte Methoden nutzen, um die bereits vorhandene Absicht in eine Tat umzusetzen. Sonst kommt es trotz ausgeprägtem Willen nicht zum nächsten Schritt.

Diese sogenannte Intentions-Verhaltens-Lücke kennen viele Menschen aus eigenen guten Vorsätzen am Jahresanfang [9]. Hier bietet sich eine Handlungs- und Bewältigungsplanung an, mit der man das Vorhaben genauer analysieren kann [10, 11, 12]. Das heißt für Lisa, sie spezifiziert mit Herrn Möchtegern die erarbeiteten Ziele anhand der SMART-Kriterien [1]. Durch genaues Festlegen seiner Übung begleitet sie ihn dabei mit den Fragen: „Wann, wo und wie (evtl. auch mit wem) möchten Sie üben?“ Herr Möchtegern soll vorausschauend überlegen, welche Hindernisse auftreten könnten und wie er diese umschiffen will. Ein solcher Plan - möglichst schriftlich notiert - wirkt nachhaltiger, wenn eine gemeinsame Entscheidungsfindung stattgefunden hat [13].

Hat Herr Möchtegern seine Übung unter Lisas Aufsicht bereits umgesetzt, kommt ihm jetzt seine Planung zugute. Diese steigert seine Selbstwirksamkeit, und er traut sich seine Aufgabe auch außerhalb der Praxis zu. Hilfreich kann dort für ihn sein, wenn er bewusst abgespeicherte physiologische Zustände wie ein Bewegungsgefühl abrufen kann und diese mit internen verbalen Verstärkungen oder Merksätzen kombiniert, zum Beispiel „die Hand schließen, sie wieder öffnen und mit dem Daumen zu jedem Finger wandern“. Eine Frage, die Lisa zusätzlich stellen kann und die Herrn Möchtegern den Verantwortungsball wieder zuspielt, ist „Was würde Ihnen helfen, diese Übung zu Hause durchzuführen?“.

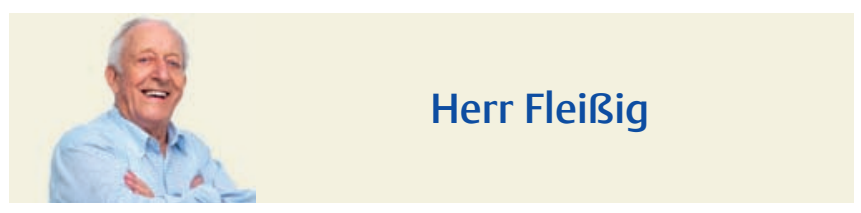

Den Erfolg halten und Rückfällen vorbeugen > Den dritten Klienten im Bunde plagen dieselben körperlichen Probleme. Bei ihm hat Lisa jedoch herausgefunden, dass Herr Fleißig nicht nur motiviert, sondern auch tatsächlich sehr engagiert seine Eigenübungen absolviert. Er befindet sich bereits im Stadium „aktiv werden“. Hier sind Strategien gefragt, welche die Aktivität aufrechterhalten, denn Herr Fleißig soll sich durch gezieltes Beobachten selbst kontrollieren lernen. Lisa bespricht mit ihm die Soll-Ist-Vergleiche seines Verhaltens. Was hat sich Herr Fleißig laut Plan vorgenommen und was davon hat er tatsächlich umgesetzt? Aus welchen Gründen gab es Abweichungen? Wie kann er diese regulieren?

Die mit „Coaching und Befähigung“ bezeichnete Phase steht für ein angewandtes (Selbst-)Coaching (॰ Abb. 1, S. 21). Die erlernten Methoden, am Ball zu bleiben, beherrscht der Klient und nutzt sie bei Bedarf. Dabei gelingt es ihm, auch störende Einflüsse alleine zu kontrollieren und mit möglichen Rückschlägen positiv und zielorientiert umzugehen. Die Therapeutin hat den Klienten damit zur Selbstbefähigung angeleitet (Empowerment).

Weg vom Frust und hin zur Eigenmotivation > Ein Verhalten im kleinen oder auch größeren Stil zu verändern ist Bestandteil jeder Therapieeinheit. Um dieses komplexe und wichtige Vorgehen greifbarer zu machen, sind individuelle Methoden nützlich, die auf einer genauen Analyse des Motivationsniveaus des Klienten basieren. Diese sogenannten Coaching-Handwerkszeuge wie spezielle Fragetechniken passen dann genau zu den Bedürfnissen des Klienten. Sie sparen einerseits Zeit und ersparen andererseits Frusterlebnisse über den „nicht kooperativen“ Klienten. Sie lassen sich schnell umsetzen und unterstützen den Klienten beim Aufblühen eigener Motivation. Die optimale Interaktion zwischen Therapeutin und Klient gewährleistet schließlich den Erfolg und die dauerhafte Umsetzung.

Nils Boettcher

\section{$\Rightarrow$ Das Literaturverzeichnis finden Sie unter www.thieme.de/ ergoonline > „ergopraxis“ > „Artikel“.}

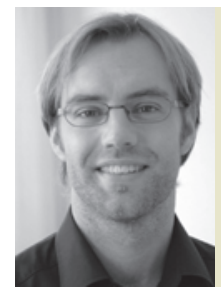

Nils Boettcher, Physiotherapeut BSc., M.A. und NLP-Lehrtrainer (DVNLP), lehrt unter anderem an der ZHAW Winterthur Kommunikation und Patientenedukation. Zudem arbeitet er in seiner eigenen Praxis mit dem Schwerpunkt Gesundheitscoaching und gibt Seminare zum beschriebenen Thema (www.emota.de). 